\title{
The MHC-Based Suppression (MBS) Theory: Implications for Transplantation
}

\author{
Derek W.R. Gray* \\ Nuffield Department of Surgery, University of Oxford, Oxford, UK
}

\begin{abstract}
Complex areas of scientific endeavor may sometimes benefit from a theoretical and/or reductionist approach to guide the direction of future experiments, perhaps best illustrated by the field of cosmology. The field of immunology in general, and transplantation immunology in particular, is certainly complex. This commentary draws attention to a theory that proposes an alternative role for MHC molecules, placing them central to the process of tolerance induction, with major implications for transplantation and all fields of immunology.
\end{abstract}

Key Words: Immune tolerance, MHC-based suppression, peptide/MHC complex, peripheral tolerance, regulatory T cells, xenotransplantation.

\section{WHAT IS KNOWN ABOUT MHC MOLECULES?}

Originally named as human leukocyte antigens (HLA), later the term major histocompatibility (MHC) antigens came into common use. In this article, the term HLA will be used to indicate the human system, whilst the term MHC will be used only to indicate a deliberate widening to include other mammalian species, all of which have a superficially similar immune system. Many of the immunological mechanisms and concepts mentioned are arguably established facts and will not be referenced, unless the point being made is of particular importance for the concepts presented here. The term "cognate ligand " is used to mean the combination of MHC and bound peptide which binds fully to a particular T cell receptor.

\section{HLA - THE HUMAN MHC}

HLA molecules have been under intensive investigation for more than 25 years and a number of remarkable features have been discovered. Firstly, there are several molecular families - HLA-A, HLA-B, HLA-C, HLA-DP, HLA-DQ, HLA-DR, HLA-E, HLA-F, HLA-G, HLA-H - that are structurally related (Ig superfamily) membrane-bound molecules with 4 immunoglobulin-like domains, an anchoring transmembrane portion, and a groove on the exterior portion of the molecule which normally has a peptide bound to it. (Further discussion will be limited to the "classical" HLA genes and their products, whereas HLA-E, HLA-F, HLA-G, HLA$\mathrm{H}$, will not be discussed further here). HLA-A, HLA-B and HLA-C form a more closely-related sub-group, called HLA Class 1 , that use a constant molecule, $\beta 2$ microglobulin, as one of the four domains. HLA-DP, HLA-DQ, HLA-DR form a second structurally-related subgroup, known as MHC Class

*Address correspondence to this author at the Nuffield Department of Surgery, John Radcliffe Hospital, Headington, Oxford OX3 9DU, UK; Tel: 44(0)1865220145; Fax: 44(0)1865768876;

E-mail: derek.gray@nds.ox.ac.uk
II, that use two different double-Ig-like molecules to produce the 4-domain structure.

Secondly, both MHC Class I and MHC Class II molecules exhibit genetically-coded (and therefore heritable) polymorphism that is so extensive that completely identical matching by non-related persons is rarely possible. Despite the polymorphism, all Type 1 and Type II MHC molecules are able to bind peptides derived from a wide range of proteins, holding them in the groove on the surface of the molecule, where they may be presented to immunocytes derived from the thymus ( $\mathrm{T}$ cells). An important advance was the demonstration that $\mathrm{T}$ cells only detect peptide when bound to an MHC molecule [1, 2], this unique arrangement being made possible by selection of $\mathrm{T}$ cells in the thymus.

\section{DEVELOPMENT OF T CELLS IN THE THYMUS}

Each $\mathrm{T}$ cell precursor (thymocyte) is produced with a $\mathrm{T}$ cell receptor (TCR) that is highly variable between individual $\mathrm{T}$ cells, and each $\mathrm{T}$ cell bears multiple identical TCR copies. The TCR variability between cells is not germ-line genetically-coded, but rather produced within each individual by a process of combined somatic mutation and alternative splicing (similar to that used to form the extreme variability seen in the hypervariable region of the immunoglobulin molecule). Initially, the developing T cells in the thymus carry two surface co-receptor molecules, named CD8 and CD4, which facilitate binding to MHC Class I and MHC Class II, respectively, but the T cell appears to choose one of the several MHC molecules displayed on thymus cortical epithelial cells (apparently at random), and the redundant coreceptor is lost. It is at this stage that positive selection occurs; only the $\mathrm{T}$ cells which bind strongly to one MHC molecule and the appropriate co-receptor are allowed proceed to the next stage of negative selection, where thymic medullary dendritic cells expose the $\mathrm{T}$ cells to diverse peptides derived from self-proteins. Strong-binding T cells are 
eliminated, presumably because, if released, the $\mathrm{T}$ cells would have the potential to initiate an auto-immune attack.

However, common sense tells us that this negative selection process cannot be $100 \%$ efficient, since we know from the Human Genome Project that humans have over 10,000 genes, each gene potentially giving rise to multiple peptides; so every $\mathrm{T}$ cell would have to be tested against every possible peptide, an impossible task. Furthermore, many proteins are post-translationally modified or expressed only in unique locations. The implication is that thymic negative selection is always incomplete, and that potentially self-reactive lymphocytes will be present in normal individuals, a logical prediction that has now been proven beyond doubt [3-5].

\section{EXPRESSION OF MHC MOLECULES}

MHC Class I is expressed, albeit sometimes at low levels, on the cell membrane of almost every nucleated cell in the body (the exception being neurons). Each MHC Class I molecule normally carries a peptide that is usually derived from processed cytosolic proteins, sized between 8 and 11 amino acids in length, limited by the size of the MHC Class I binding groove, which is closed at both ends.

MHC Class II molecules are not normally expressed on most cells, except for those that are considered to be professional antigen-presenting cells (APCs), which are dendritic cells, macrophages, monocytes and B cells. However, an important fact (not universally understood) is that $\mathrm{MHC}$ Class II is rapidly expressed (within 6 hours) on almost all cells in response to a number of stimuli, but most powerfully in response to gamma interferon production, itself an early accompanying feature of most cellular immune events. The peptides presented by MHC Class II molecules on APCs are thought to be derived from mainly external proteins taken up by a pinocytotic mechanism, processed to peptides, and loaded in the MHC groove. The peptide size is more varied than in the case of MHC Class I, probably because the binding groove is open at one end: 9-25 amino acids would be typical.

\section{T CELL ACTIVATION}

The discriminatory sensitivity of the T cell-peptide-MHC interaction is remarkable. Techniques for measuring $\mathrm{T}$ cell response to "best fit" peptide/MHC (hereafter termed "cognate ligand") in vitro have steadily improved, and each new assay has found the demonstrated limit of detection lowered $[6,7]$; it now looks possible (although yet to be repeated and proven) that, under normal in vivo conditions, a $\mathrm{T}$ cell response to cognate ligand may even be driven by a $\mathrm{T}$ cell detecting a single peptide located in the groove of a single MHC molecules [8].

\section{ACTIVATION OF NAÏVE VERSUS ANTIGEN-EXPE- RIENCED T CELLS}

One important point which is perhaps the source of much confusion is that there is a considerable difference between the signals required to activate naive $\mathrm{T}$ cells, where costimulatory molecules are mandatory for full activation, and those for antigen-experienced $\mathrm{T}$ cells, often called armed effector $\mathrm{T}$ cells, which do not require co-stimulation for activation. The activation of naïve $\mathrm{T}$ cells normally takes place mostly in lymphoid tissue (lymph nodes and spleen), mostly in lymphoid tissue (lymph nodes and spleen), whereas armed effector cells target "peripheral tissues" (by which are meant all tissues other than lymphoid tissue, any of which may be subject to attack by an invader or a selfpeptide), as the normal site for the $\mathrm{T}$ cell decision. The timing and specificity of the response of $\mathrm{T}$ cells is very different in the two circumstances.

\section{THE MOLECULAR MECHANISM OF T CELL AC- TIVATION IS NOT UNDERSTOOD: EARLIER MOD- ELS WERE SIMPLISTIC}

Until recently, the TCR binding to $\mathrm{MHC} /$ peptide complex was viewed as a simple single phase "on-off " binding event, and attempts were made to correlate TCR affinity and binding on and off-rates onto peptide/MHC complexes with ability to activate $\mathrm{T}$ cells. Despite earlier claims to the contrary, it has not been possible to develop a repeatable measure of the binding event that predicts the $\mathrm{T}$ cell response $[9$, 10], there are always "exceptions" and it has become evident that a wide variety of influences (cytokines, costimulatory molecules, etc) alter $\mathrm{T}$ cell responses. The concept of "tuning" has been raised, which arguably means just unexplained variability. It is even the case that a completely novel mechanism for $\mathrm{T}$ cell activation could be plausible. For example, a two-stage binding process and recognition of $\mathrm{MHC}$ as a "super-dimer" has recently been proposed [11, 12], with convincing evidence presented.

\section{INHIBITORY MECHANISMS IN T CELL CONTROL}

The description so far given for the process of $\mathrm{T}$ cell activation would probably be agreed as accepted fact by most immunologists, but there is another side to $\mathrm{T}$ cell activity which is mandated by the point made above regarding incomplete thymic deletion of potentially self-reactive $\mathrm{T}$ cells. Incomplete thymic deletion means that every time a $\mathrm{T}$ cell recognizes cognate ligand there is the possibility that this could be derived from either a foreign invader or a selfprotein; if it is the former the $\mathrm{T}$ cell must activate. If it is the latter, then the $\mathrm{T}$ cell must be prevented from activation and clonal expansion. Getting it right is crucial, since death may be the outcome if the immune system makes the wrong choice. Our understanding of how this inhibition is achieved is complicated by there being a number of apparently separate mechanisms used, including ignorance, deletion, anergy, and regulation (previously called suppression) [13]. Recent investigation and comparison of anergy and induced regulatory cell formation is difficult to combine or summarize, as the systems and reagents used have varied considerably, but the one constant feature is the need for MHC/peptide contact [14-20]. Regulatory cells may be produced in the thymus and in the periphery, and can arise from conversion of non-inhibitory $\mathrm{T}$ cells [21-23]. There is increasing evidence to support the view that it is the activation status of the $\mathrm{T}$ cell at the point of receiving an inhibitory signal that dictates the response (the nature of this signal will be discussed later). Naive $\mathrm{T}$ cells tend to respond to inhibitory signaling by becoming anergic, and this is reversible [17] whilst armed effector cells respond to the same stimuli by either apoptosis or, if they survive, then become active regulatory cells, able to inhibit a new influx of primed antigen-specific cells; this state is not reversible [17]. 
The origin of these clearly inhibitory phenomena is currently suggested to arise from $\mathrm{MHC} /$ cognate ligand/TCR engagement in the absence of danger signals. The latter now form a long list, including Toll-like receptor signaling, certain chemokines and cytokines, including IL-1 $(\alpha$ and $\beta)$, IL2 , IL-6, IL-17, and costimulatory signals, such as those transmitted by ligand/receptors, such as CD28-CD80/86, CD40CD154 and ICOS-ICOS ligand.

The concept of co-inhibition was introduced more than 10 years ago [24, 25], and has slowly expanded. The term now includes signaling via CD152-mediated inhibition of CD28, by competitive binding of CD80/CD86, signaling via PD1/PD1 ligand, and production of inhibitory cytokines, such as IL 10 and TGF-beta. These are considered the extracellular molecules that mediate inhibition spreading to other $\mathrm{T}$ cells. The transcription factor Foxp3 is part of, and acts as a marker for, the intracellular inhibitory pathways associated with acquisition of regulatory function, and the downstream pathways leading to multiple gene activation have been identified.

It is suggested here that most of the control mechanisms described above are delivered mainly via migratory dendritic cells acting on naive $\mathrm{T}$ cells within lymphoid tissue. In contrast, the $\mathrm{T}$ cell decision in the peripheral tissues is usually taken by armed effector $\mathrm{T}$ cells contacting cognate ligand peptide presented on MHC molecules in peripheral tissues, and here understanding the role of the MHC becomes crucial.

\section{WHAT IS THE PURPOSE OR ADVANTAGE OF THE OBLIGATORY BINDING OF PEPTIDES TO MHC FOR T CELL RECOGNITION, AND WHY IS THE MHC SO POLYMORPHIC?}

The interpretation of the role of MHC molecules is important for our understanding of the immune system. The extreme variability of the MHC is conserved in all mammalian species (but is most extensively developed in humans) and is unique in this respect amongst the many gene systems in the mapped human genome. The currently accepted explanation for the polymorphism of HLA is that it ensures that, in the event of a new pathogenic organism developing, which has a protein structure that is totally novel, there will be at least one or more individuals with MHC molecules able to bind the novel foreign peptides to the MHC binding groove. This could be termed the pathogen adaptation approach, and is supported by a number of disease-association studies (see [26] for a good summary). This explanation places the $\mathrm{MHC} /$ peptide/T cell interaction at the heart of $\mathrm{T}$ cell activation, but gives it no active role in inhibitory responses.

However, there are conceptual difficulties about the dogma that the fundamental purpose of the MHC polymorphism is to ensure new invaders are recognized and dealt with. First, why is there a difference from the B cell, which is the other major lymphocyte population in the mammalian immune system that has as its main purpose the capability to detect foreign protein, but achieves this by expressing B cell receptors which are highly variable within each individual? Although polymorphism of HLA is extreme within the human population, each individual has only 12 classical MHC molecule variants, which have to presumably bind many millions of different peptides throughout our lifetime. Obviously, peptide binding must be highly promiscuous, a point which has only been fully recognized and proven to be the case quite recently [27].

Keeping this point in mind, and recognizing that the number of different peptides that would be seen as invaderderived is likely to be large for most invasive organisms, it would seem likely that HLA variation will, in practice, have little effect on the ability of most individuals to respond to a whole range of organisms, a fact demonstrated by the longevity of humans (at least in comparison to most species). Of course, there have been major pandemics when millions have died, but it is suggested here that the ability of the survivors of those events to deal with the offending organism was more likely to be a result of the diversity of the $\mathrm{T}$ cell and $\mathrm{B}$ cell receptors within each individual (and their ability to detect multiple invader peptides and expand armed effector cells) rather than the variation of HLA within their population, allowing efficient peptide binding.

The finding that inbred farm and laboratory animals show MHC-related differences in immune response to certain organisms has sustained the dogma for years, but does not prove that, outside the special inbred circumstance, this is the main driving force for the polymorphism of HLA. In keeping with this argument (with the possible exception of the cytotoxic T lymphocyte response in HIV infection) there has been no striking evidence of patients with restricted HLA phenotypes preferentially surviving epidemic infections; indeed, most of the variability of response to human infection is unrelated to the MHC [28]. So, if invader peptide binding is not the main selection mechanism driving $\mathrm{MHC}$ polymorphism, what is?

\section{DEVELOPMENT OF THE MHC-BASED SUPPRES- SION HYPOTHESIS}

It was pointed out nearly 10 years ago [29] that, if one tried to produce a biological system for preventing HLA matching between individuals, it would be difficult to develop anything more effective than the HLA gene complex; So maybe that is exactly what it is doing - not, one assumes, to prevent organ transplantation, but to protect some crucial function from masquerade, presumably by invading organisms. This alternative explanation for the polymorphism of HLA views the system as providing the equivalent of an individual security code, but exactly what is it protecting?

A strong clue must lie within the known function of HLA. T cells presumably spend most of their lifespan constantly binding and unbinding their TCRs onto thousands of peptide/MHC complexes, always alert for contact with cognate ligand peptide. When that event arrives, as discussed above, the $\mathrm{T}$ cell must discriminate between an invaderderived peptide and a self-peptide, a crucial decision on which may rest the survival of the individual. If the decision is that the peptide is invader-derived, a full immune response must be launched, but if self-derived then the immune response must be switched off. The molecular basis of this decision almost certainly corresponds to the mechanism of peripheral tolerance, the identity of which has eluded researchers in autoimmunity and transplantation for over 25 years. 
However, one factor that has not been taken into account in previous studies is the risk that this switch-off mechanism presents to the entire species. If one invading organism was able to duplicate the signal, then, because the $\mathrm{T}$ cell is the central controller of cellular and humoral responses (and possibly innate responses too [30]), the individual concerned would be virtually defenseless. Furthermore, if a limited number of invariant molecules were the basis of the switchoff mechanism throughout the species, then mimicry by an invader could put the whole species at risk. How likely would that scenario be? One has only to look at the problem of antibiotic-resistance in the last 50 years to see how rapidly pathogenic organisms can overcome defense strategies.

The point is that this decision must be made secure from invader duplication or mimicry by a mechanism which must be unique (or nearly so) for each individual, and only be translatable by that one individual. In a mammalian biological system the only conceivable way to do this is via a molecular system with high inter-individual variability. The recent mapping of the human genome makes it clear there is only one candidate gene system with sufficient variability and that is the MHC gene complex. It is therefore not likely to be a coincidence that MHC molecules are purposedesigned to carry the very peptides that are the subject of the $\mathrm{T}$ cell decision. Is the logical argument presented above sufficient to justify a paradigm shift at this point in time? Fig. (1) summarizes the decision balance as it seems to this author, favoring the change, but readers must make up their own minds.

The central proposal of the theory presented here is that the main purpose of the extreme polymorphism of HLA is to protect the $\mathrm{T}$ cell decision-making mechanism by preventing invaders from being able to produce molecules that could masquerade as switch-off signals. For this to be possible a

\section{MHC polymotphism - what is its purpose?}

consequential mechanism must be proposed: $\mathrm{T}$ cell contact with self-MHC must produce a signal, separate and distinct from the activation signal delivered by MHC/peptide. This signal guides the $\mathrm{T}$ cell decision between invader and selfcognate ligand peptide, and is an absolute requirement for suppression/regulatory mechanisms. Recognizing the central function of the MHC in the process, the term MHC-based suppression (MBS) was adopted. The fundamental concept is shown in Fig. (2).

\section{EXTENDING THE HYPOTHESIS INTO A FULLY DEVELOPED MODEL}

Because the approach of logic combined with analysis of a wide survey of the published literature (as described above) appeared to have delivered a novel concept, the same approach was undertaken to extend the hypothesis into a fullydeveloped model incorporating most relevant areas of immunology. The full details of the literature found, the reasoning behind the logic chain, and the mechanisms by which the MBS signal is used to guide $\mathrm{T}$ cell homeostasis, activation and inhibition are described in a main treatise and most will not be detailed here. The treatise has been placed as an openaccess web-based publication: Etreatises in biology \& medicine, ISSN1753-2027, URL: http://etreatises.net). This form of publication has the added advantage of being simple to update with a new version whilst storing an archive of all previous versions. To future-proof the publication, a permanent open-access repository has also been put in place in the Oxford Research Archive (http://ora.ouls.ox.ac.uk:8081/ 10030/989).

Obviously, the mechanism central to the MBS theory must explain how the MHC/peptide/TCR interaction can produce two signals instead of the one currently recognized. The proposed model and publications that are particularly

\section{MHC molecules bear the peptides which are the subject of the T cell decision}

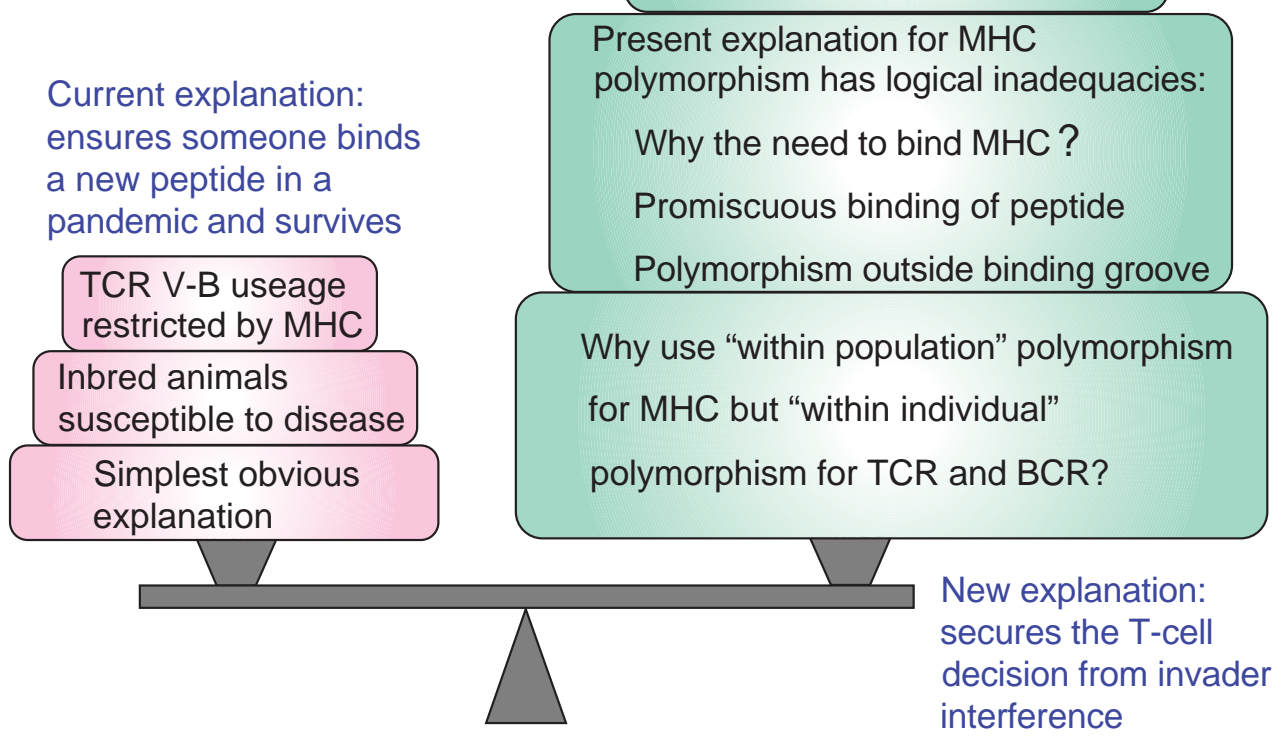

Fig. (1). An imaginary "decision balance" summarizing the evidence for the current and proposed new explanations accounting for the extreme polymophism of MHC molecules. The block sizes represent the author's view on the relative strength of each argument. 


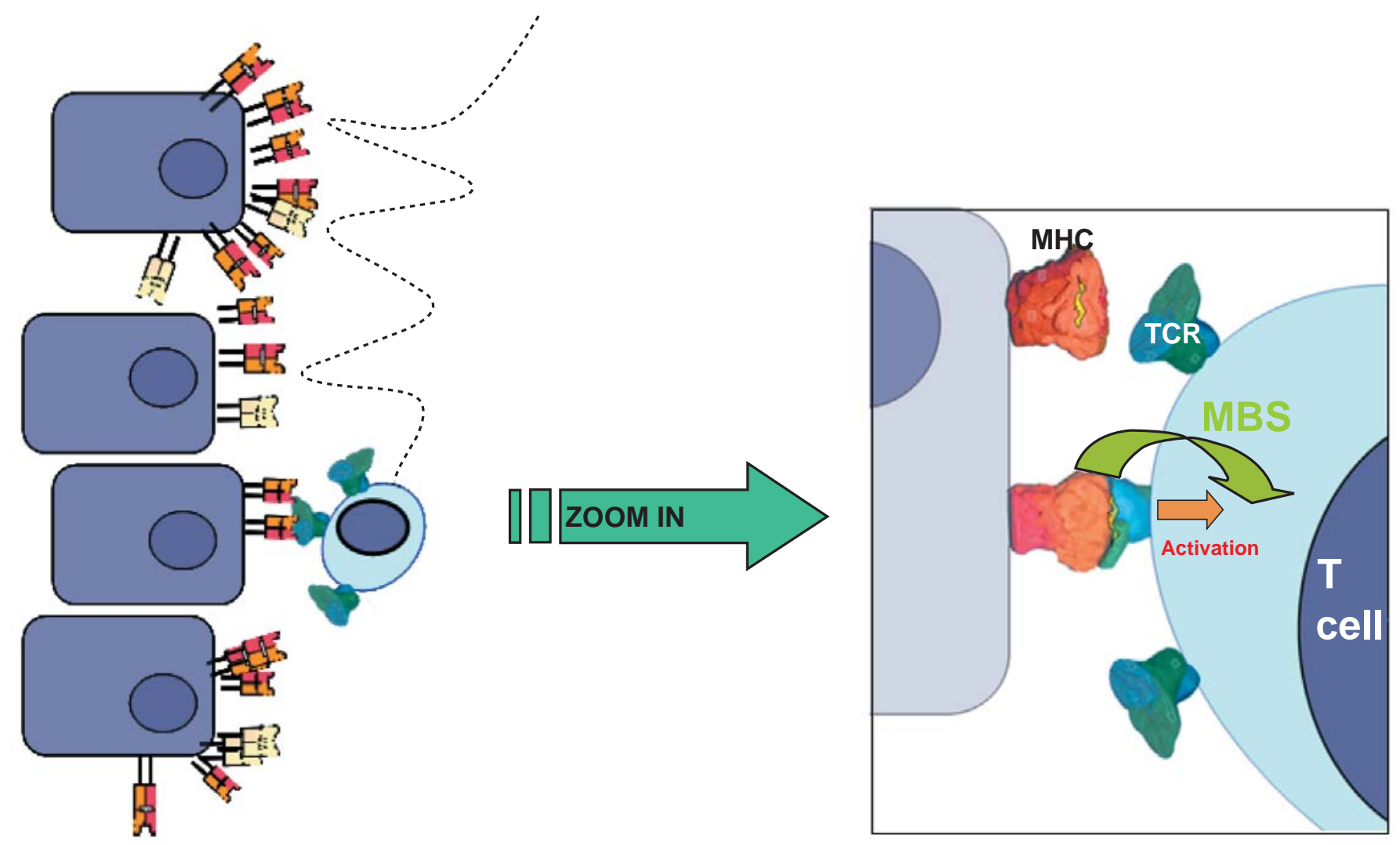

Fig. (2). A cartoon representation of the fundamental concept behind the MHC based suppression (MBS) hypothesis.

supportive will be briefly presented here in the hope that it will interest readers sufficiently to visit the etreatises website, where more detail can be found.

\section{A PROPOSED MOLECULAR MECHANISM FOR GENERATING A MHC-BASED SUPPRESSION SIG- NAL}

It is clear that the MBS signal must be derived from the $\mathrm{TCR} /$ peptide/MHC interaction in the same event as $\mathrm{T}$ cell activation signaling, and is not a separate event. There are a limited collection of molecules that have extensive experimental evidence to support their involvement in either or both signaling processes, and these are shown in Fig. (3A).

Fig. (3B) shows the current view of the physical arrangement of these molecules and the pathways involved in $\mathrm{T}$ cell signaling that produce $\mathrm{T}$ cell activation. The greyedout molecules are those that are known to influence $\mathrm{T}$ cell activation, but no exact role or mechanism has been identified: they include CD4 (or CD8), CD45, and the phosphorylated protein kinases Csk, Fyn and Lyn.

It should be noted that the role of the co-receptor (CD4 or CD8) in T cell signaling is not well understood, and T cell activation can occur in its absence (but requires much higher ligand concentrations). The most striking unexplained feature of both molecules is the long extracellular extension of both CD4 and CD8 molecules, the tips displaying an expanded binding motif which normally binds to highly conserved sites on the membrane-proximal part of the bound MHC molecules.

\section{THE LINK BETWEEN T CELL ACTIVATION, CD4 OR CD8 AND CD45}

Numerous studies have described a role for the protein tyrosine phosphatase CD45 in determining $\mathrm{T}$ cell signaling thresholds (see [31] for summary). CD45 deficient mice fail to develop a normal immune system and $\mathrm{T}$ cell development in the thymus is arrested at the double positive (CD4 plus CD8) stage, the signaling deficit being corrected by Lck "knock-in" [32], showing the importance of CD45 and it's relationship to the activation phosphorylation cascade. Antibody cross-linking studies established functional connections between CD4 and CD45 [33]. Specifically, Odum et al. concluded that, in activated human $\mathrm{T}$ cells, class II signals are up regulated by $\mathrm{CD} 4$, which is associated with p56lck, and down regulated by $\mathrm{CD} 45$, which is a tyrosine phosphatase having the ability to influence the activation threshold of $\mathrm{T}$ cells through Fyn/Lyn signaling [33] . The obvious approach for identifying the MBS pathway was, therefore, to incorporate the above molecules which appeared to be difficult to fit into the presently-accepted model of $\mathrm{T}$ cell activation pathway.

Figs. (3C and 3D) show the molecules (in cartoon form) that constitute the proposed, essentially inhibitory, MBS signal, and their likely arrangement.

The fundamentals of the proposed mechanism can be more easily grasped by reducing it to key components, as shown in Figs. (4A-D). It has been termed the mechanical signaling model because it effectively measures the mechanical "fit" of the TCR/peptide/MHC complex by triangulating 


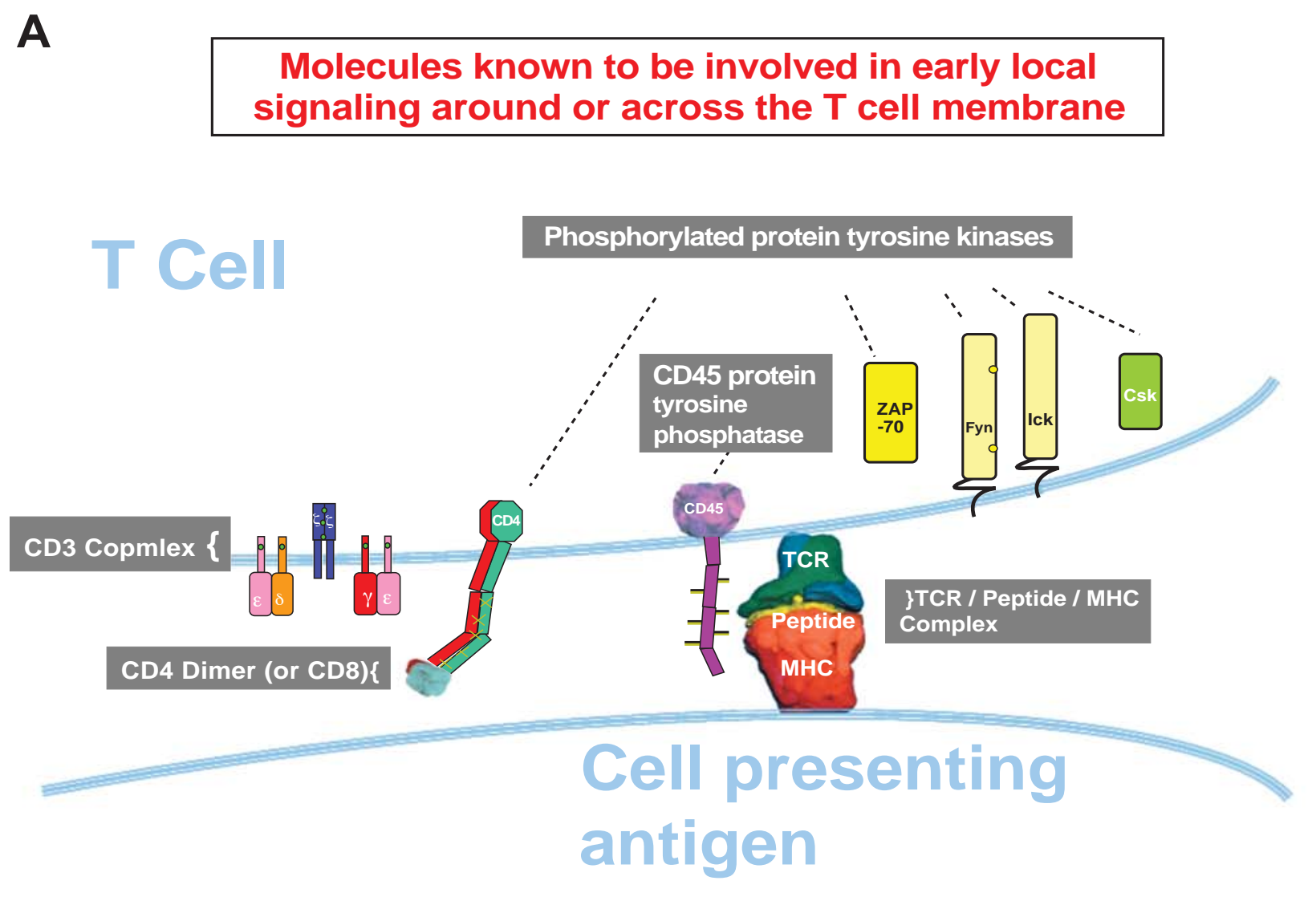

B

CD3 conformational change promotes activation

ITAMS loaded, then drive ZAP-70 phosphorylation

TCR/peptide/MHC binding:

Affinity low

Binding kinetics unusual

Minimal conformational change

No constant predictor of activation identified despite intensive search

Integrated protein

tyrosine kinase cascade 
(Fig. 3) contd....
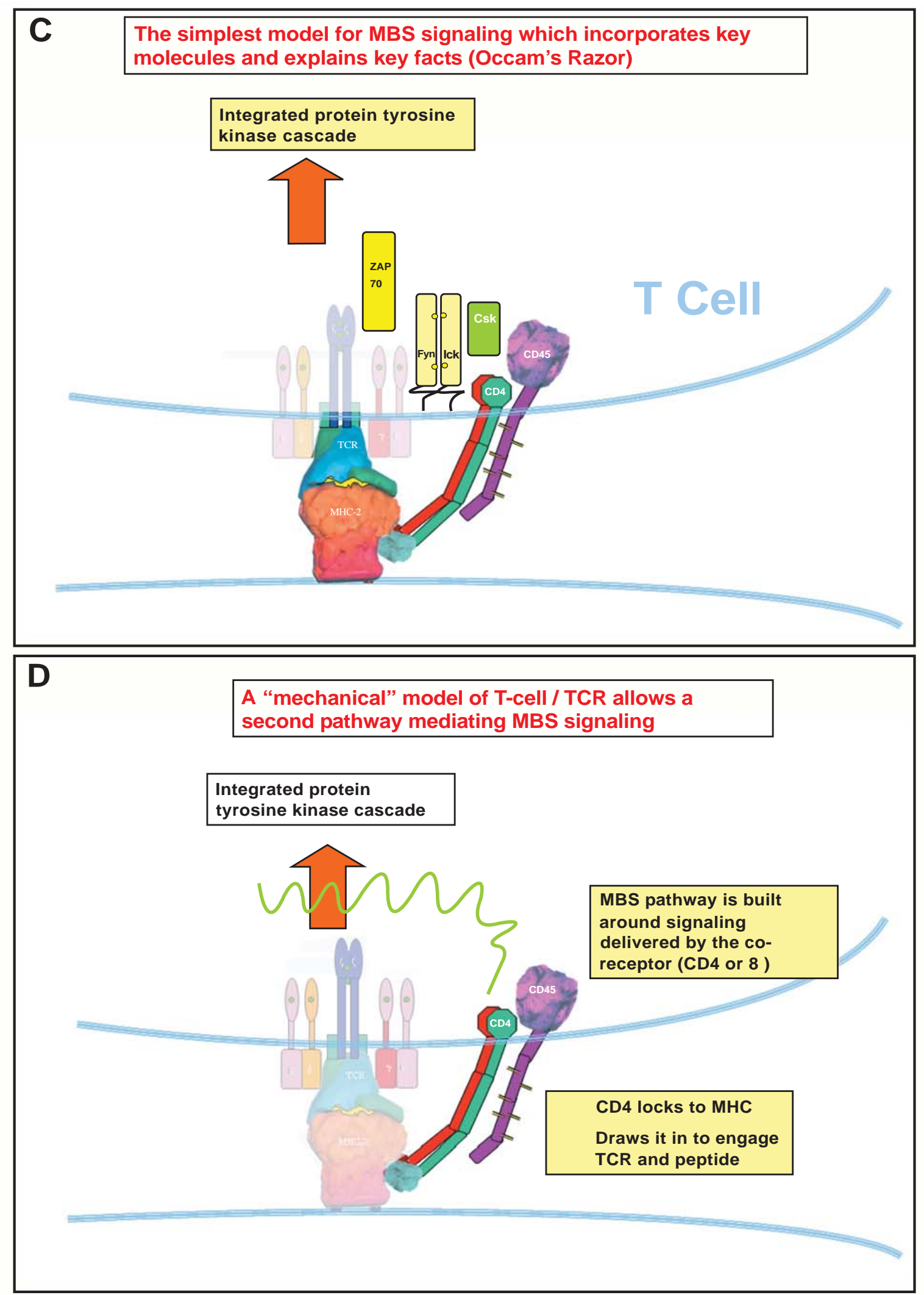

Fig. (3). A. A cartoon representation of the molecules in and around the cell surface that are likely to have a role in early signaling between cells presenting (MHC/peptide) antigen and T cells receiving signal. B. Diagram to show the author's interpretation of the currently-favored molecular pathway for a cell presenting antigen to signal $\mathrm{T}$ cell activation. C. Diagram to show the author's suggestion for the molecular pathway for the hypothesised MHC-based suppression signal. D. A simplified version of 2C emphasising the key role of CD4 (CD8) as a mechanical sensor of MHC/TCR engagement 
A

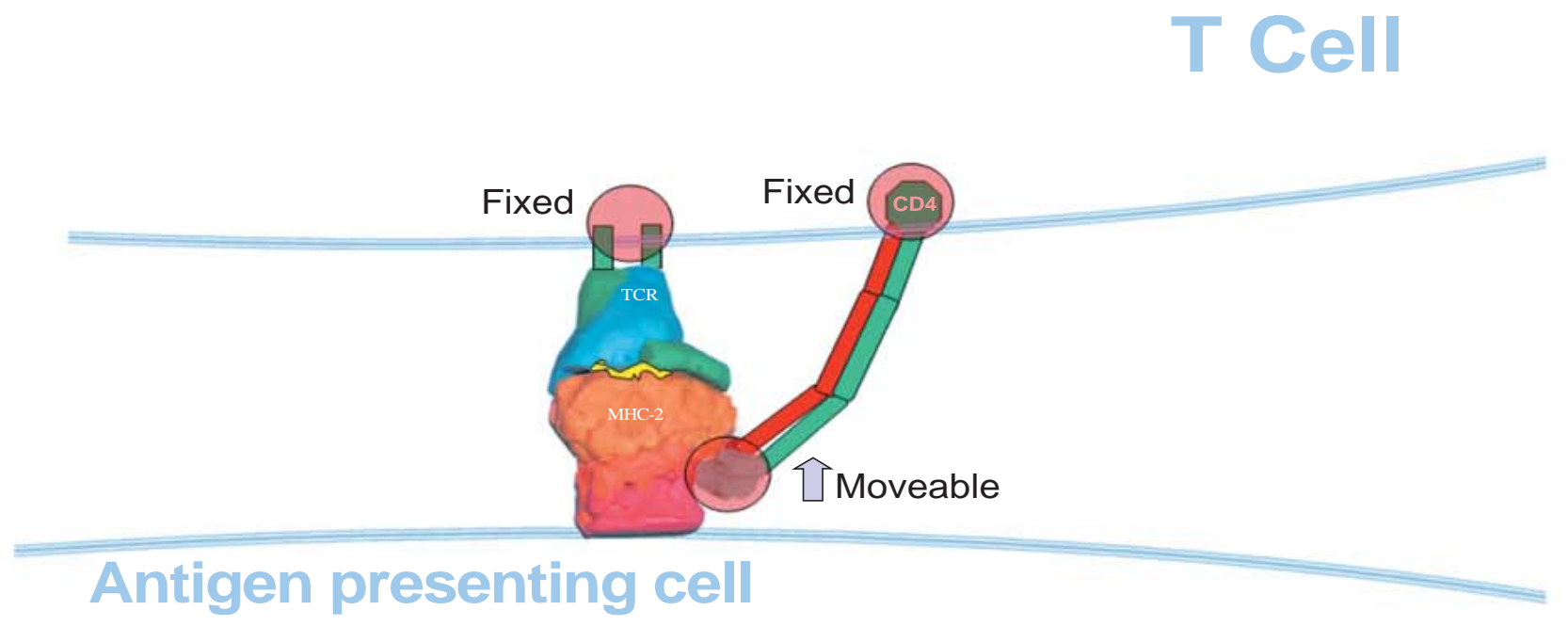

B

This distance (arrowed) corresponds to the extent of TCR/MHC engagement allowed by the bound peptide
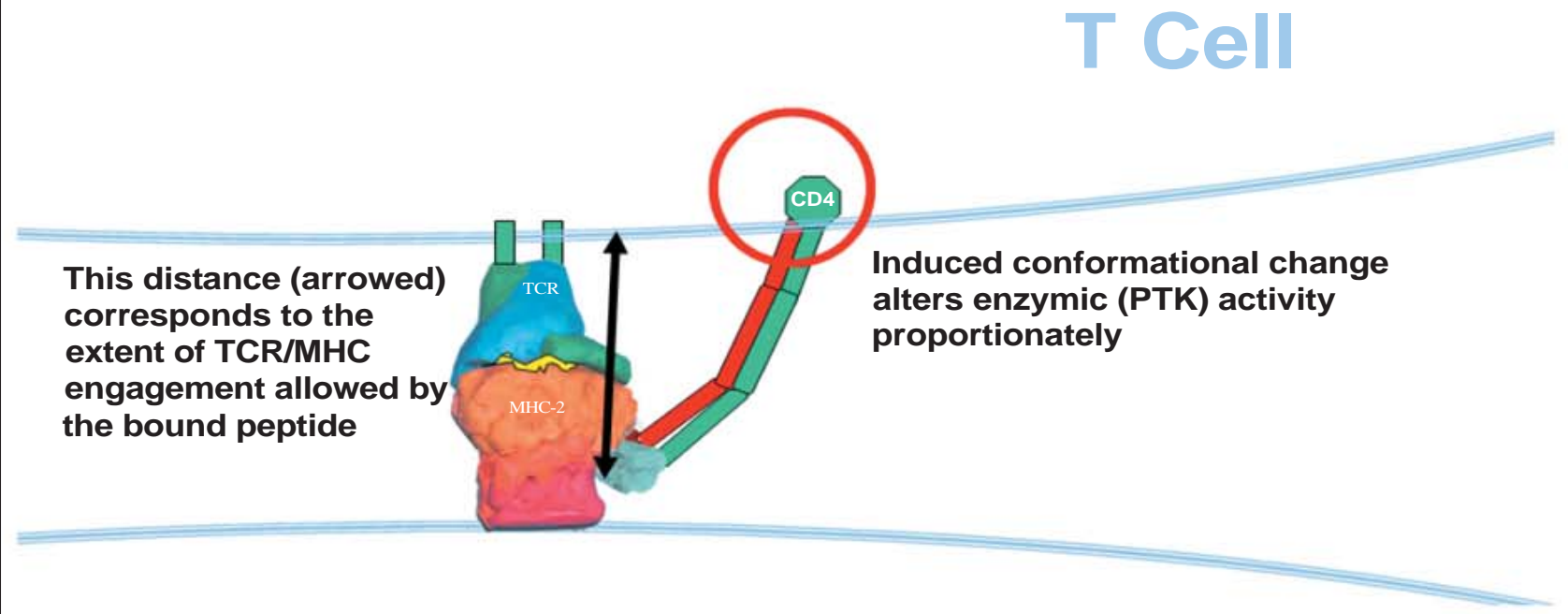
(Fig. 4) contd....

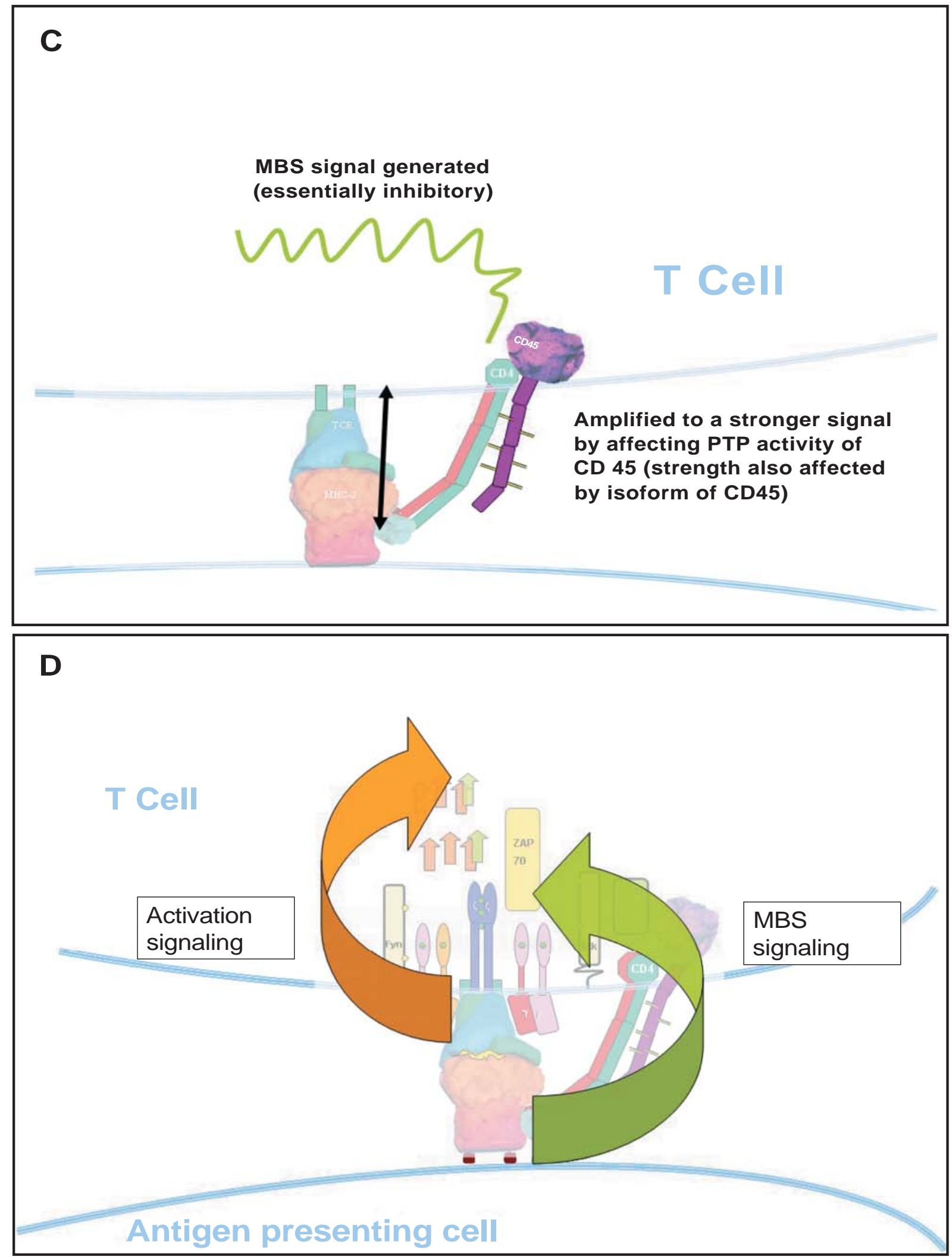

Fig. (4). A. Diagram showing the underlying principle of two anchor points and one moveable point. B. Diagram showing how the mechanical model produces signal. (PTK = protein tyrosine kinase). C. Diagram showing the proposed role of CD45 for amplifying and converting the signal to inhibitory by increased phosphatase activity from the intracellular phosphatase enzyme domain. The effect of the delivered inhibitory MBS signal is to raise the threshold for T cell activation, promoting enhanced specificity. (PTP = protein tyrosine phosphatase). D. Diagram emphasizing the reciprocal relationship of the two signals arising from MHC/peptide/TCR engagement, which are subsequently integrated via a final common pathway. 
three points - Point 1 is the trans-membrane portion of the TCR, Point 2 is the trans-membrane portion of CD4/8 in the $\mathrm{T}$ cell membrane, and Point 3 is the CD4/8 binding site on the presented MHC molecule. The first two points are fixed, but the third point is grasped by the ectodomain of CD4/8 and drawn into the binding site as far as the bound peptide will allow. The deformation and conformational change is passed to the intracellular enzyme domain of CD4/8, altering its enzymic phosphorylation activity, which in turn affects the much more powerful phosphatase activity of CD45, which therefore acts as an amplifier of the MBS signal.

The mechanism proposed has been chosen as the simplest explanation fitting the known characteristics of molecules that affect $\mathrm{T}$ cell activation. An initial active primary binding process of MHC to CD4/8 would explain the slower than expected binding association that has been found for TCR/peptide/MHC. It would also fit as the first step in the recently proposed two-step binding process [12]. Furthermore, it is possible that full engagement of the TCR by the $\mathrm{MHC} /$ peptide may result in locking of the CD4/8 attachment, giving a prolonged signal and slow "off" binding. This would greatly enhance the activation signaling, accounting for the thousand-fold increase in sensitivity associated with co-receptor expression. Lastly, if full engagement does not occur, it may be the case that the disengagement of the coreceptor then acts as a "quick release" mechanism to free the $\mathrm{TCR}$, ready to move to the next MHC/peptide for testing.

\section{EXPERIMENTAL FINDINGS THAT STRONGLY SUPPORT THE EXISTENCE OF MBS}

\section{Targeted Mutations of TCR, MHC and Altered Peptide Ligands}

The structure and full genetic sequence is now available for several individual MHC / peptide / TCR combinations and techniques for site directed mutagenesis of individual amino acids (aa) within these molecules have become relatively straightforward. By using well-defined systems that normally show $\mathrm{T}$ cell activation, the effect of individual mutations on the strength of activation can be assessed. A large number of such studies were carried out in the 1990s, looking usually at the effect of substituting single aa's within one or two components such as the TCR or peptide. Many different experimental systems were used, making exact comparisons between studies difficult, but the overall findings were consistent and applied to both CD4 and CD8 T cells. They are summarized in Figs. (5A-B), (with representative references cited in the Figure) [34-38].

Two points of importance for the MBS theory arise from these studies. The first finding was that single aa substitutions affecting binding areas of the MHC molecule outside the binding groove and to a lesser extent the groove sidewalls produced a marked increase in $\mathrm{T}$ cell activation. This increase was accompanied by a reduction in peptide specificity, broadening the peptide spectrum that would initiate $\mathrm{T}$ cell activation. These characteristics are similar to those seen in the response of $\mathrm{T}$ cells to contact with allogeneic $\mathrm{MHC}$, where there are typically 6-10 aa differences from syngeneic MHC molecules, and approximately $1-10 \%$ of $\mathrm{T}$ cells are activated versus the normal 1 in 100,000 (approx) $\mathrm{T}$ cells that respond to a typical syngeneic $\mathrm{MHC}$ bearing a novel peptide [39]. Many ingenious mechanisms have been proposed to explain the latter finding, but the MBS theory gives the simplest explanation, which is that single aa substitutions affecting binding areas of the MHC molecule outside the binding groove and to a lesser extent the groove sidewalls reduce MBS signaling, which in turn lowers the threshold for $\mathrm{T}$ cell activation.

The second finding from aa substitution was that single amino acid changes to an activating peptide may produce a so-called "altered peptide ligand" that can have properties ranging from strong agonist, to strong antagonist, the latter being even capable of preventing $\mathrm{T}$ cell activation by the original peptide/MHC $[40,41]$. The antagonist effect is seen when both the original peptide and the antagonist peptide are presented together, and has been shown to require both peptides to be MHC-bound (i.e. direct binding competition between peptides has been excluded). To explain this finding it has been necessary to invoke a new phenomenon named as "Specific MHC/peptide blocking", [40] or "TCR antagonism" [41], no further detailed mechanism being so far described. It is proposed here that these are effectively alternative terms for MHC-based suppression, and the phenomenon is in fact the best proof of the existence of MBS,

Recently a direct and simple approach of using antiMHC antibody to block regulatory cell action has been reported in both CD4 and CD8 T cell regulatory systems [20, 42], the finding being that regulatory function in both systems was completely blocked, results compatible with MBS,

\section{HOW IS IT ENVISAGED THAT THE MBS INHIBI- TORY SIGNAL IS USED WITHIN THE MAMMAL- IAN IMMUNE SYSTEM?}

The problem the immune system has is how to distinguish self peptides from invader peptides in a manner that cannot be interfered with by an invader. It is proposed that repeated contact of the T $\mathrm{C}$ R with self MHC delivers a continuous stream of reliable, non-duplicatable "reassurance signal" which effectively holds the T cell under continuous inhibitory suppression: MHC-based suppression.

The MBS signal is proposed to have a central role in several $\mathrm{T}$ cell functions (listed in Table $\mathbf{1}$ and discussed in turn below).

Table 1. How is MBS Inhibitory Signal Used ?

1) Thymic selection

2) Preventing $\mathrm{T}$ cells from be coming activated whilst circulating

3) T cell homeostasis

4) The $T$ cell choice after binding cognate ligand

5) Regulatory cell function

\section{1) Thymic Selection of $T$ Cells}

\section{i) Positive Selection}

It is proposed that positive selection of T-cells in the thymus initializes and sets the "normal gain and threshold" of the MBS signal for each T cell, when binding its selected 

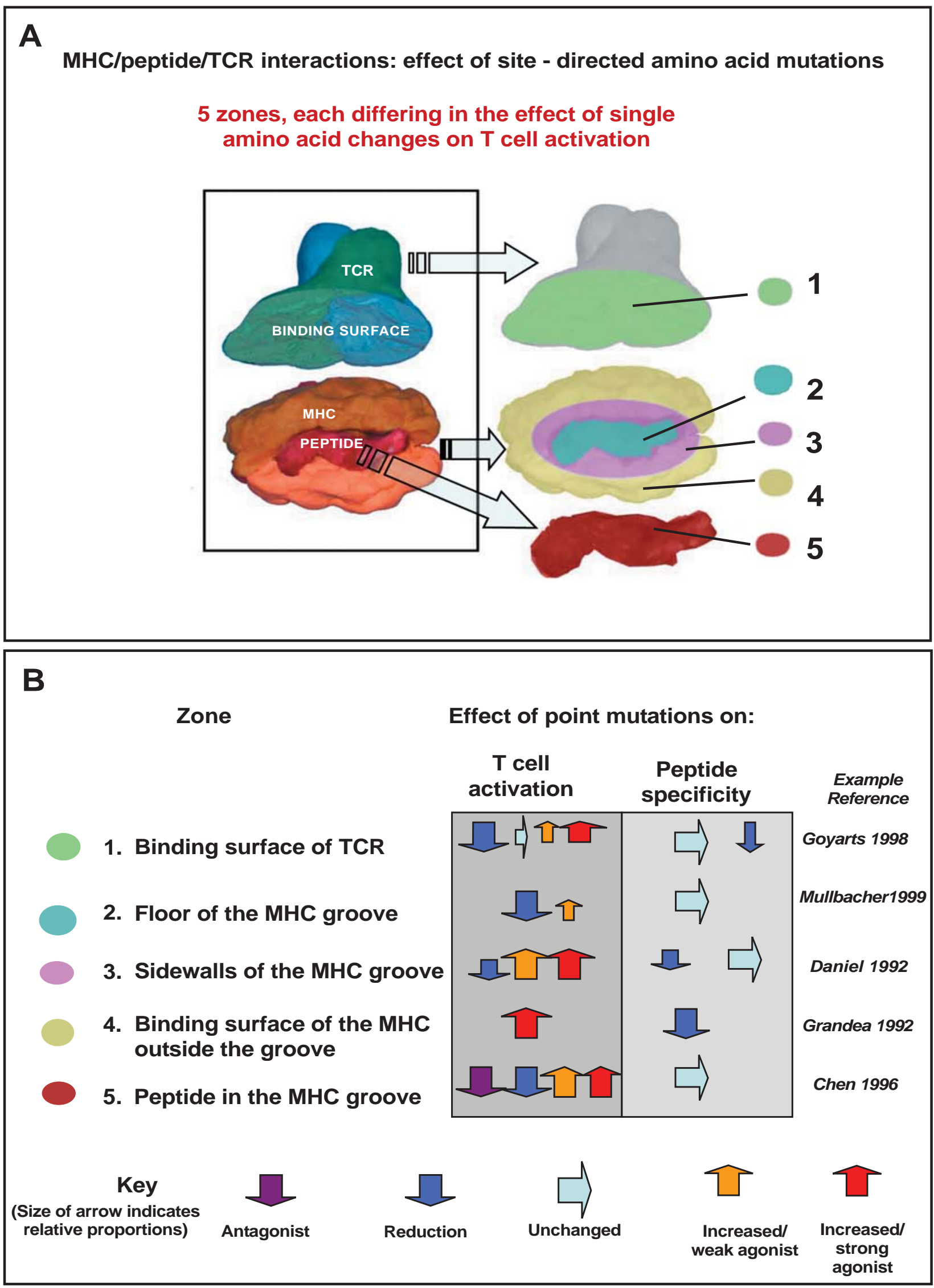

Fig. (5). A. Cartoon diagram illustrating the binding surfaces of the TCR/MHC/peptide interaction that have been investigated by sitedirected single amino acid mutagenesis. 5 zones have been identified and are shown diagrammatically. B. Tabulation to show the effect of single amino acid mutations in each zone on $\mathrm{T}$ cell activation and peptide specificity. 
MHC molecule. Importantly, the peptide(s) carried during the positive selection process would be MHC-derived, thus making the future MBS signal maximal when those peptides are in the MHC groove. Note also that the pattern of peptides inducing maximum MBS signal varies because the TCR for each $\mathrm{T}$ cell undergoing the positive selection process varies, so that each $\mathrm{T}$ cell has an individual "peptide signature" that delivers maximum MBS signal to its own TCR or to derived $\mathrm{T}$ cell clones.

\section{ii) Negative Selection}

MBS signaling is then used immediately by the $\mathrm{T}$ cell to examine peptide/MHC complexes displayed on intrathymic antigen presenting cells, the outcome of cognate ligand binding in the presence of sufficient MBS signal in the thymus being ensures that the deletion process is secure from intrathymic invader attack.

\section{2) Preventing $\mathbf{T}$ Cells from Becoming Activated Whilst Circulating}

It is logically important that circulating $\mathrm{T}$ cells are not activated until they reach their target and a prime candidate to perform this function would be soluble MHC, which has been shown to be suppressive to autologous $\mathrm{T}$ cells in vitro and in vivo, yet no accepted role in vivo has so far been demonstrated. Again the mechanism would be repeated contact of the TCR of one $\mathrm{T}$ cell contacting the MHC bearing self peptide/MHC on other $\mathrm{T}$ cells with sufficient frequency that the MBS signal is enough to maintain suppression.

\section{3) T Cell Homeostasis}

If the number of $\mathrm{T}$ cells available for contact within the lymphoid tissue drops the MBS signal produced will reduce and if it drops below a critical level in the absence of cognate ligand then several rounds of homeostatic proliferation are undergone, continuing until the MBS signal rises above the threshold once again.

\section{4) The T Cell Choice: Activation Versus Inhibition after Binding Cognate Ligand}

\section{i) Differences between Nä̈ve T Cells and Antigen Experi- enced T Cells}

The role of MBS differs between the first recognition of cognate ligand by naïve $\mathrm{T}$ cells (which normally occurs in the lymphoid tissue) and the later contact of antigen experienced $\mathrm{T}$ cells (alternatively called armed effector cells) which normally takes place in the peripheral tissues). Naïve $\mathrm{T}$ cells maintain a high activation threshold from their constant repeated contact with $\mathrm{MHC} /$ peptide delivering MBS signal and require high levels of activation signal from cognate ligand, which is usually assured by the costimulatory function of molecules such as CD 86 on the antigen presenting cell (usually a dendritic cell) binding to CD28 on the lymphocyte coincident with TCR engagement. This activation sequence may only be stopped by a powerful MBS signal, such as that delivered by contact with regulatory cells (see below).

The product of the primary immune response is clonal expansion and the production of armed effector cells which, when mature, circulate between lymphoid tissue and the pe- ripheral tissues. In the periphery they may be caused to leave the circulation by the expression of adhesion molecules at the site of invader attack. Within the tissues the arriving armed effector T cells may encounter MHC bearing cognate ligand once again, and this time the threshold for activation is lower and there is no need for co-stimulation. It is proposed that the initial recognition event when the armed effector T cell TCR binds cognate ligand MHC/peptide complex produces signaling through the TCR/CD3 complex as currently accepted and does not primarily involve MBS signaling. Then follows the crucial point when the decision between invader and self peptide must be taken.

\section{ii) Proposed Mechanism for Distinguishing Invader from Self Peptide: T Cells Responding to a Self Peptide will be a Singleton Clone}

The binding and recognition of cognate ligand causes the $\mathrm{T}$ cell to enter a "sampling mode" for 6-12 hours, and it is the events taking place during this delay which are the most important and currently ill-understood factors controlling $\mathrm{T}$ cell responses. It is suggested that during this period the receptors of the $\mathrm{T}$ cell in question make contact with multiple MHC/peptide molecules on adjacent cells, accumulating MBS signal from self MHC bearing self peptides, and integrating co-stimulatory and co-inhibitory signals. The accumulation of MBS signal would be most powerful from self MHC bearing the same self MHC-derived signature peptide set as was originally used to set up the MBS signal during thymic selection. Any $\mathrm{T}$ cell derived from the same clone will get the same maximum inhibitory signal from contacting the same $\mathrm{MHC} /$ peptide set and will deliver maximum inhibition to other T-cells of the same clone by expressing the same MHC/peptide set. T cells from a different clone will not get the same inhibitory signal. The net effect will be to switch to suppression when the $\mathrm{T}$ cell is responding to a singleton peptide (as will likely be the case for an auto-reactive $\mathrm{T}$ cell) but to continue to activation when the response is to multiple peptides, indicating an invader.

\section{iii) Metabolic Changes Necessary for Activation Continue through the Sampling Period}

Whilst the events above proceed during the sampling phase following ligand engagement, the metabolism of the responding $\mathrm{T}$ cell is progressively altered, in preparation for effector cell function. If the inhibitory threshold is not reached then activation will follow, with features that are well characterized involving a protein tyrosine kinase cascade. If the accumulating MBS signal crosses the inhibitory threshold (which was initially set in the thymus) then the activation process stops, the fate of the affected T lymphocyte then being dependant upon how far down the differentiation track it has gone. With increasing length of time before the threshold is reached the response to this inhibitory switch goes from reversible anergy to non-reversible anergy to regulatory cell formation and finally to $\mathrm{T}$ cell apoptosis.

\section{5) Regulatory T Cells also Use MBS}

Once a T cell has "made the decision" that the cognate ligand detected is from a self protein, the $\mathrm{T}$ cell response must shift from attack to protection of the cell bearing that ligand. The logical mechanism for this to occur would be for the $\mathrm{T}$ cell to stop migrating and simply express high levels of 
MHC loaded with "signature" MHC-derived peptides (achieved by producing gamma interferon locally), making sure that any new $\mathrm{T}$ cells (from the same clone) arriving receive a strong MBS signal to prevent activation.

\section{IMPLICATIONS FOR TRANSPLANTATION}

As already stated the MBS theory provides an attractive explanation for the direct presentation alloresponse, in which up to $10 \%$ of allogeneic $\mathrm{T}$ cells may respond to contact with allo-MHC by becoming activated. MBS theory interprets this phenomenon as arising from $\mathrm{T}$ cell contact with allogeneic MHC/peptides, which deliver an activation signal, but are unable to deliver the full amount of MBS signal, and so the activation threshold (and the specificity) falls. Only the indirect presentation pathway would be able to generate sufficient MBS inhibitory signal to switch the active $\mathrm{T}$ cells from activation to suppression. It is therefore of some importance that nearly all active regulatory cell systems analysed have indeed found that regulation works via the indirect pathway (and the exceptions are explicable)[43, 44].

If the MBS theory is correct then the implications for xenotransplantation in particular are considerable. From the "point of view" of the immune system, a xenograft will fit the description of an invader trying to mimic the MBS signaling system, bearing barely recognizable MHC molecules that deliver a severely-reduced MBS signal, and having numerous amino acid differences in every protein which, on processing by APCs, will generate large numbers of peptides that can induce an indirect response. The prediction is that producing peripheral tolerance to xenogeneic tissue or organs by conventional immunosuppression alone will be very difficult, if not impossible. Replacing porcine MHC with common human MHC alleles will help by restoring the MBS signaling, but the sheer number of peptide differences will still drive a vigorous response, and therefore some sort of (? extracorporeal) selective removal of reactive $\mathrm{T}$ cells will also be required.

\section{CONCLUSIONS}

This commentary has argued for a re-interpretation of the purpose and role of MHC polymorphism, for which the author believes the logic is inescapable and supported by considerable evidence, albeit circumstantial. Such a paradigm shift fits the known features of transplantation well. To encourage investigators to produce relevant data from experiments that would address the issue, a proposed molecular mechanism is presented. It is, of course, unlikely to be accurate in all aspects, but it is hoped that it will provide an inspiration to others to think outside the current dogma. Attention is again drawn to a website (eTreatises in biology \& medicine, ISSN1753-2027, URL: http://etreatises.net). That contains a treatise summarizing the current evidence, which will shortly be updated with extensive additions.

\section{ABBREVIATIONS}
$\mathrm{APC}=$ Antigen-presenting cell
HLA $=$ Human leukocyte antigens
MHC $=$ Major histocompatibility complex
$\mathrm{TCR}=\mathrm{T}$ cell receptor

\section{REFERENCES}

[1] Doherty PC, Zinkernagel RM. H-2 compatibility is required for Tcell-mediated lysis of target cells infected with lymphocytic choriomeningitis virus. J Exp Med 1975; 141: 502-7.

[2] Quill H, Carlson L, Fox BS, et al. Optimization of antigen presentation to $\mathrm{T}$ cell hybridomas by purified Ia molecules in planar membranes. Ia molecule polymorphism determines the antigenic fine specificity of the response to cytochrome c peptides. J Immunol Methods 1987; 98: 29-41.

[3] Fowell D, Mason D. Evidence that the T cell repertoire of normal rats contains cells with the potential to cause diabetes. Characterization of the CD4+ T cell subset that inhibits this autoimmune potential. J Exp Med 1993; 177: 627-36.

[4] Lohmann T, Leslie RD, Londei M. T cell clones to epitopes of glutamic acid decarboxylase 65 raised from normal subjects and patients with insulin-dependent diabetes. J Autoimmun 1996; 9: 385-9.

[5] Topliss D, How J, Lewis M, et al. Evidence for cell-mediated immunity and specific suppressor $\mathrm{T}$ lymphocyte dysfunction in Graves' disease and diabetes mellitus. J Clin Endocrinol Metab 1983; 57: 700-5.

[6] Demotz S, Grey H, Sette A. The minimal number of class II MHCantigen complexes needed for T cell activation. Science 1990; 249: 1028-30.

[7] Reay PA, Matsui K, Haase K, et al. Determination of the relationship between $t$ cell responsiveness and the number of MHC-peptide complexes using specific monoclonal antibodies. J Immunol 2000; 164: 5626-34.

[8] Irvine DJ, Purbhoo MA, Krogsgaard M, et al. Direct observation of ligand recognition by T cells. Nature 2002; 419: 845-9.

[9] Davis SJ, Ikemizu S, Evans EJ, et al. The nature of molecular recognition by T cells. Nat Immunol 2003; 4: 217-24.

[10] Rudolph MG, Stanfield RL, Wilson IA. How TCRs bind MHCs, peptides, and coreceptors. Annu Rev Immunol 2006; 24: 419-66.

[11] Krogsgaard M, Li QJ, Sumen C, et al. Agonist/endogenous peptide-MHC heterodimers drive $\mathrm{T}$ cell activation and sensitivity. Nature 2005; 434: 238-43.

[12] Wu LC, Tuot DS, Lyons DS, et al. Two-step binding mechanism for T-cell receptor recognition of peptide MHC. Nature 2002; 418: 552-6.

[13] Choi S, Schwartz RH. Molecular mechanisms for adaptive tolerance and other T cell anergy models. Semin Immunol 2007; 19: $140-52$.

[14] Van Rensen AJ, Taams LS, Grosfeld-Stulemeyer MC, et al. Induction of $\mathrm{T}$ cell anergy by liposomes with incorporated major histocompatibility complex (MHC) II/peptide complexes. Pharm Res 2000; 17: 720-6.

[15] Witzke O, Wheeler PR, Barbara JA, et al. Suppression mediated by anergic $\mathrm{CD} 4+\mathrm{T}$ cells requires stimulation by MHC-peptide complexes and can be induced in the presence of costimulation. Transplant 2001; 72: 369-76.

[16] Jiang S, Camara N, Lombardi G, et al. Induction of allopeptidespecific human $\mathrm{CD} 4+\mathrm{CD} 25+$ regulatory $\mathrm{T}$ cells ex vivo. Blood 2003; 102: 2180-6.

[17] Knoechel B, Lohr J, Zhu S, et al. Functional and molecular comparison of anergic and regulatory T lymphocytes. J Immunol 2006; 176: 6473-83.

[18] Nishimura E, Sakihama T, Setoguchi R, et al. Induction of antigenspecific immunologic tolerance by in vivo and in vitro antigenspecific expansion of naturally arising Foxp3+CD25+CD4+ regulatory T cells. Int Immunol 2004; 16: 1189-201.

[19] Taams LS, Akbar AN. Peripheral generation and function of CD4+CD25+ regulatory $\mathrm{T}$ cells. Curr Top Microbiol Immunol 2005; 293: 115-31.

[20] Peiser M, Becht A, Wanner R. Antibody blocking of MHC II on human activated regulatory $\mathrm{T}$ cells abrogates their suppressive potential. Allergy 2007; 62: 773-80.

[21] Chen W, Jin W, Hardegen N, et al. Conversion of peripheral CD4+CD25- naive $\mathrm{T}$ cells to CD4+CD25+ regulatory $\mathrm{T}$ cells by TGF-beta induction of transcription factor Foxp3. J Exp Med 2003; 198: $1875-86$.

[22] Pillai V, Karandikar NJ. Human regulatory T cells: A unique, stable thymic subset or a reversible peripheral state of differentiation? Immunol Lett 2007; 114: 9-15. 
[23] Kretschmer K, Heng TS, Von Boehmer H. De novo production of antigen-specific suppressor cells in vivo. Nat Protoc 2006; 1: 65361.

[24] Sinclair NR, Anderson CC. Co-stimulation and co-inhibition: equal partners in regulation. Scand J Immunol 1996; 43: 597-603.

[25] Truong W, Hancock WW, Anderson CC, et al. Coinhibitory T-cell signaling in islet allograft rejection and tolerance. Cell Transplant 2006; 15: 105-19.

[26] Slev PR, Potts WK. Disease consequences of pathogen adaptation. Curr Opin Immunol 2002; 14: 609-14.

[27] Engelhard VH, Bullock TN, Colella TA, et al. Antigens derived from melanocyte differentiation proteins: self-tolerance, autoimmunity, and use for cancer immunotherapy. Immunol Rev 2002; 188: $136-46$

[28] Hill AV. Aspects of genetic susceptibility to human infectious diseases. Annu Rev Genet 2006; 40: 469-86.

[29] Gray DW. Major histocompatibility complex-based suppression: a mechanism for T-cell control. Med Hypotheses 1998; 50: 289-302.

[30] Kim K, Zhao J, Auh S, et al. Adaptive immune cells temper initial innate responses. Nat Med 2007; 13: 1248-52.

[31] Hermiston ML, Xu Z, Weiss A. CD45: a critical regulator of signaling thresholds in immune cells. Annu Rev Immunol 2003; 21: 107-37.

[32] Seavitt JR, White LS, Murphy KM, et al. Expression of the p56(Lck) Y505F mutation in CD45-deficient mice rescues thymocyte development. Mol Cell Biol 1999; 19: 4200-8.

[33] Odum N, Martin PJ, Schieven GL, et al. Signal transduction by HLA-DR is mediated by tyrosine kinase(s) and regulated by CD45 in activated T cells. Hum Immunol 1991; 32: 85-94.

[34] Goyarts EC, Vegh Z, Kalergis AM, et al. Point mutations in the beta chain CDR3 can alter the $T$ cell receptor recognition pattern on an MHC class I/peptide complex over a broad interface area. Mol Immunol 1998; 35: 593-607.
[35] Mullbacher A, Lobigs M, Yewdell JW, et al. High peptide affinity for MHC class I does not correlate with immunodominance. Scand J Immunol 1999; 50: 420-6.

[36] Daniel C, Horvath S, Allen PM. A basis for alloreactivity: MHC helical residues broaden peptide recognition by the TCR. Immunity 1998; 8: 543-52.

[37] Grandea AG, Bevan MJ. A conservative mutation in a class I MHC molecule outside the peptide binding groove stimulates responses to self peptides. J Immunol 1993; 151: 3981-7.

[38] Chen YZ, Matsushita S, Nishimura Y. Response of a human T cell clone to a large panel of altered peptide ligands carrying single residue substitutions in an antigenic peptide: characterization and frequencies of TCR agonism and TCR antagonism with or without partial activation. J Immunol 1996; 157: 3783-90.

[39] Suchin EJ, Langmuir PB, Palmer E, et al. Quantifying the frequency of alloreactive T cells in vivo: new answers to an old question. J Immunol 2001; 166: 973-81.

[40] De Magistris MT, Alexander J, Coggeshall M, et al. Antigen analog-major histocompatibility complexes act as antagonists of the T cell receptor. Cell 1992; 68: 625-34.

[41] Peiser M, Becht A, Wanner R. Antibody blocking of MHC II on human activated regulatory $\mathrm{T}$ cells abrogates their suppressive potential. Allergy 2007; 62: 773-80.

[42] Joetham A, Takeda K, Miyahara N, et al. Activation of naturally occurring lung $\mathrm{CD} 4(+) \mathrm{CD} 25(+)$ regulatory $\mathrm{T}$ cells requires CD8 and MHC I interaction. Proc Natl Acad Sci USA 2007; 104: 1505762.

[43] Rulifson IC, Szot GL, Palmer E, et al. Inability to induce tolerance through direct antigen presentation. Am J Transplant 2002; 2: 5109.

[44] Sánchez-Fueyo A, Domenig CM, Mariat C, et al. Influence of direct and indirect allorecognition pathways on CD4+CD25+ regulatory T-cell function in transplantation.Transplant Int 2007; 20: 534-41.

(C) Derek W.R. Gray; Licensee Bentham Open.

This is an open access article licensed under the terms of the Creative Commons Attribution Non-Commercial License (http://creativecommons.org/licenses/ by-nc/3.0/) which permits unrestricted, non-commercial use, distribution and reproduction in any medium, provided the work is properly cited. 